\title{
Semiconductor Device Noise Computation Based on the Deterministic Solution of the Poisson and Boltzmann Transport Equations
}

\author{
ALFREDO J. PIAZZA* and CAN E. KORMAN
}

Department of Electrical Engineering and Computer Science, The George Washington University, Washington, DC 20052

\begin{abstract}
Numerical simulation results of noise due to current fluctuations along an $n^{+}-n-n^{+}$ submicron structure are presented. The mathematical framework is based on the interpretation of the equations describing electron transport in the semiclassical transport model as stochastic differential equations (SDE). According to this formalism the key computations for the spectral density describing the noise process are reduced to a special initial value problem for the Boltzmann transport equation (BTE). The algorithm employed in the computation of the space dependent noise autocovariance function involves two main processes: the stationary self-consistent solution of the Boltzmann and Poisson equations, and a transient solution of the BTE with special initial conditions. The solution method for the BTE is based on the Legendre polynomial method. Noise due to acoustic and optical scattering and the effects of nonparabolicity are considered in the physical model.
\end{abstract}

Keywords: Stochastic differential equations, Boltzmann transport equation, noise, Legendre polynomials

\section{INTRODUCTION}

Current noise in semiconductors is due to the inherent randomness of the scattering mechanisms that govern electronic transport. These current fluctuations around a stationary value are generally characterized by the associated autocovariance function or equivalently, by their spectral density. Employing the machinery of SDE, a new noise model [1] shows that the key computations for the noise autocovariance function are reduced to the transient solution of the BTE with special initial conditions. This novel approach was previously utilized [2] for the computation of the noise spectral density in bulk silicon. The BTE was deterministically solved using the Legendre polynomials method and the results were in excellent agreement with those obtained using the Monte

\footnotetext{
* Corresponding author.
} 
Carlo technique. In this paper we study the impact that the spatial variation of the doping has on the current noise autocovariance function. The subject of our study is a one-dimensional $n^{+}-n-n^{+}$ submicron structure in the stationary regime. The outline of our paper is as follows. Sections II and III concentrate on the noise and physical models respectively. Section VI describes the algorithm and Section V presents the numerical results. The last section is devoted to the conclusions.

\section{THE NOISE MODEL}

According to semiclassical transport theory, the motion of an electron in a semiconductor is described by the following stochastic differential equations:

$$
\begin{gathered}
\frac{d \vec{x}}{d t}=\vec{v}(\vec{k})=\frac{1}{\hbar} \nabla_{\vec{k}} \varepsilon(\vec{k}), \quad d \frac{\vec{p}}{d t}=\hbar \frac{d \vec{k}}{d t}=-q \vec{E}+\vec{F}_{r}, \\
\text { and } \quad \vec{F}_{r}=\sum_{i} \hbar \vec{u}_{i} \delta\left(t-t_{i}\right)
\end{gathered}
$$

where $\vec{x}, \vec{v}, \vec{p}$ and $\vec{k}$ are the electron position, velocity, momentum and wave vector, respectively, $\vec{E}$ is the electric field, $\varepsilon(\vec{k})$ is the energy-wave vector relationship in the given energy band and $\vec{F}_{r}$ is the random impulse force on the electron due to scattering. The random force is characterized by the transition rate $W\left(\vec{k}, \vec{k}^{\prime}\right)$. Accordingly, the probability of scattering is given by

$$
\begin{aligned}
\operatorname{Pr}\left\{t_{i}-t_{i-1}>\tau\right\} & =\exp \left\{-\int_{t_{i-1}}^{t_{i-1}+\tau} \lambda\left(\vec{k}\left(t^{\prime}\right)\right) d t^{\prime}\right\}, \\
\lambda(\vec{k}) & =\int W\left(\vec{k}, \vec{k}^{\prime}\right) d \vec{k}^{\prime}
\end{aligned}
$$

where $\lambda(\vec{k})$ is the scattering rate. Therefore, given the electron wave vector $\vec{k}, \lambda(\vec{k}) \Delta t$ is the probability that a jump in momentum will occur in a small time interval $\Delta t$. Assuming that a scattering event has occurred at some time $t_{i}$, the probability density function for the amplitude of the jump is given by,

$$
\chi_{\vec{k}_{i}}\left(\vec{u}_{i}\right)=\frac{W\left(\vec{k}_{i}, \vec{k}_{i}+\vec{u}_{i}\right)}{\lambda\left(\vec{k}_{i}\right)}
$$

where $\overrightarrow{k_{i}}=\vec{k}\left(t_{i}^{-}\right)$and $\vec{k}_{i}+\vec{u}_{i}=\vec{k}\left(t_{i}^{+}\right)$.

These same equations describe the electron motion in Monte Carlo simulations.

In the context of the SDE theory, these equations correspond to a Markov process, which is characterized by a transition probability function satisfying the Kolmogorov-Feller equation. In the case of semiclassical transport the latter equation is identical to the linear (non-degenerate) BTE (eq. 4)

$$
\begin{array}{r}
\frac{\partial \rho}{\partial \tau}\left(\vec{x}^{\prime}, \vec{k}^{\prime}, 0 ; \vec{x}, \vec{k}, \tau\right)+\vec{v}(\vec{k}) \cdot \nabla_{\vec{x}} \rho- \\
\frac{q}{\hbar} \vec{E}(\vec{x}, \tau) \cdot \nabla_{\vec{k}} \rho= \\
\int \rho\left(\vec{x}^{\prime}, \vec{k}^{\prime}, 0 ; \vec{x}, \vec{k}^{\prime \prime}, \tau\right) W\left(\vec{k}^{\prime \prime}, \vec{k}\right) d \vec{k}^{\prime \prime}-\lambda(\vec{k}) \rho
\end{array}
$$

subject to the following initial condition:

$$
\left.\rho\left(\vec{x}, \vec{k}^{\prime}, 0 ; \vec{x}, \vec{k}, \tau\right)\right|_{\tau=0}=\delta\left(\vec{k}-\vec{k}^{\prime}\right) \cdot \delta\left(\vec{x}-\vec{x}^{\prime}\right),
$$

where $\delta(\cdot)$ is the Dirac delta function and the following notation has been adopted:

$$
\rho\left(\vec{x}, \vec{k}, \tau \mid \vec{x}^{\prime}, \vec{k}^{\prime}, 0\right) \equiv \rho\left(\vec{x}^{\prime}, \vec{k}^{\prime}, 0 ; \vec{x}, \vec{k}, \tau\right)
$$

Generally, noise in semiconductors is characterized by the spectral density of current fluctuations. The spectral density is defined as the Fourier transform of the autocovariance function. The autocovariance of any random process can be found from the transition probability density function of such random process. Since the transition probability function satisfies the Kolmogorov-Feller equation, and the BTE is identical to it, the transition probability density function can be obtained from the solution of the BTE subject to appropriate initial conditions.

In [1] it was shown that the current longitudinal noise autocovariance function can be obtained 
from the transient solution of the BTE subject to the following "special" initial condition:

$$
\left.\rho(\vec{x}, \vec{k}, \tau)\right|_{\tau=0}=\left(v(\vec{k})-\langle\vec{v}\rangle_{\vec{x}}\right) f(\vec{x}, \vec{k}) .
$$

where

$$
\langle\vec{v}\rangle_{\vec{x}} \equiv \int v(\vec{k}) f(\vec{x}, \vec{k}) d \vec{k}
$$

Here, in (7) $f(\vec{x}, \vec{k})$ represents the steady state solution of the BTE. The current autocovariance function is computed

$$
K_{\vec{J}}(\vec{x}, \tau)=q^{2} \int v(\vec{k}) \rho(\vec{x}, \vec{k}, \tau) d \vec{k}, \quad \tau \geq 0 .
$$

It is very important to note that this approach for the current noise autocorrelation computation is strictly within the framework of semiclassical transport. This approach directly connects the physics of scattering with the current noise characteristics and makes no additional assumptions regarding the nature of the noise.

\section{THE DEVICE MODEL}

A device under stationary conditions is appropriately characterized by the Poisson Eq. (10) and the space-dependent BTE (11). The self-consistent solution of these equations provides $f(\vec{x}, \vec{k})$, the stationary probability density function and $\phi(\vec{x})$, the electrostatic potential throughout the device. In mathematical form these equations are given by:

$$
\nabla_{\vec{x}}^{2} \phi(\vec{x})=\frac{q}{\varepsilon_{s}}[n(\vec{x})-N(\vec{x})] .
$$

where $N(\vec{x})$ is the doping concentration and $n(\vec{x})=\int f(\vec{x}, \vec{k}) d \vec{k}$ is the free charge concentration and

$$
\begin{array}{r}
\vec{v}(\vec{k}) \cdot \nabla_{\vec{x}} f(\vec{x}, \vec{k})-\frac{q}{\hbar} \vec{E}(\vec{x}) \cdot \nabla_{\vec{k}} f= \\
\int f\left(\vec{x}, \vec{k}^{\prime \prime}\right) W\left(\vec{k}^{\prime \prime}, \vec{k}\right) d \vec{k}^{\prime \prime}-\lambda(\vec{k}) f
\end{array}
$$

Since in our example we are using a $1-D n^{+}-n-$ $n^{+}$structure, $\vec{E}$ and $\vec{x}$ are parallel and can be replaced by the scalars $E$ and $x$. It is also assumed that $\vec{E}$ is in the symmetric [ $\left[\begin{array}{lll}1 & 1 & 1\end{array}\right]$ crystallographic direction and therefore a single band distribution function accurately represents the state of the momentum space.

\section{THE ALGORITHM}

The space-dependent noise auto-correlation function is computed according to the following straight-forward procedure:

1. Obtain $f(x, \vec{k})$ and $\phi(x)$ from the self consistent solution of the Poisson Eq. (10) and spacedependent BTE (11). The self-consistent solution to the Poisson and Boltzmann equations is achieved by concurrently solving the corresponding discretized equations in a Gummeltype iteration. This process comprises the following steps:

(a) Generate initial values for $\phi(x)$ and $n(x)$ using the Drift-Diffusion model.

(b) Generate initial values for $f(x, \vec{k})$ consistent with $\phi(x)$ and $n(x)$ calculated in step (a), solving the homogeneous BTE.

(c) Obtain $f^{i+1}(x, \vec{k})$ from $f^{i}(x, \vec{k})$ solving the space-dependent BTE until the current along the device converges. The BTE is solved using the electric field that corresponds to $\phi^{i}(x)$.

(d) Solve Poisson Eq. (10) for $\phi^{i+1}(x)$ given $n^{i+1}(x)$.

(e) Steps (c) and (d) are repeated until the whole system converges.

2. Compute special initial condition $\left.\vec{g}(x, \vec{k}, \tau)\right|_{\tau=0}$ as prescribed by (7).

3. Obtain $\vec{g}(x, \vec{k}, \tau)$ solving the transient BTE (4) with the special initial condition computed in step 2, and the electric field $E(x)$ corresponding to $\phi(x)$ obtained in step 1 . The transient solution to the BTE is based on implicitly approximating the time derivative in Eq. (4). 
Therefore $g\left(x, \vec{k}, \tau^{k+1}\right)$ can be computed from the solution of Eq. (4) and the knowledge of $g\left(x, \vec{k}, \tau^{k}\right)$, for any $k$. This process is comprised of the following steps:

(a) Generate initial guess values for $g\left(x, \vec{k}, \tau^{k+1}\right)$ $=g\left(x, \vec{k}, \tau^{k}\right)$

(b) Obtain $g^{i+1}\left(x, \vec{k}, \tau^{k+1}\right)$ from $g^{i}\left(x, \vec{k}, \tau^{k+1}\right)$ solving the transient BTE, Eq. (4), until total current converges.

(c) Advance the time index and go to step (a).

4. Compute the autocovariance function according to Eq. (9).

\section{SOLUTION OF THE BTE EMPLOYING LEGENDRE POLYNOMIALS}

In order to map ellipsoidal energy surfaces into spherical ones, the Herring-Vogt transformation is employed. In this transformation the original coordinates $\vec{x}, \vec{E}$ and $\vec{k}$ are mapped into $\vec{x}^{*}, \vec{E}^{*}$ and $\vec{k}^{*}$, respectively and the dispersion relationship becomes spherical $\gamma(\varepsilon)=\varepsilon+\beta \varepsilon^{2}=\hbar^{2} k^{* 2} /$ $2 m_{0}$ in the new domain. The BTE remains unchanged in the stared variables and the direction of $\vec{E}^{*}$ defines a symmetry axis $(\hat{z})$. The density function dependence on momentum can be expressed in terms of only two independent variables: $\varepsilon$ and $\theta$. We expand $f\left(x^{*}, \vec{k}^{*}, t\right)$ in Legendre polynomials according to:

$$
\begin{aligned}
f\left(x^{*}, \vec{k}^{*}, t\right)= & f_{0}\left(x^{*}, \varepsilon, t\right)+k^{*} g\left(x^{*}, \varepsilon, t\right) \cos \theta \\
& +k^{* 2} h\left(x^{*}, \varepsilon, t\right)(3 \cos 2 \theta-1) .
\end{aligned}
$$

This representation for $f\left(x^{*}, \vec{k}^{*}, t\right)$, is replaced in Eq. (4). Recalling that the Legendre polynomials are orthogonal, the resulting equation is solved for the corresponding coefficients independently. This results in a system of three coupled differentialdifference equations for the functions $f_{0}, g$ and $h$, in terms of $t, x^{*}$ and $\varepsilon$. The system is solved for $f_{0}\left(x^{*}, \varepsilon, t\right)$ in the energy domain using standard finite difference method. The functions $g\left(x^{*}, \varepsilon, t\right)$ and $h\left(x^{*}, \varepsilon, t\right)$ are then obtained from $f_{0}\left(x^{*}, \varepsilon, t\right)$.

\section{NUMERICAL RESULTS}

Figure 1 shows the parameters employed in the simulation: A Gaussian doping profile ranging from $10^{16}$ to $10^{17} \mathrm{~cm}^{-3}$ with the highest doping at the device boundaries and minimum in the middle, 2 Volts of applied voltage, a device length of 0.5 $\mu \mathrm{m}$, etc. Figure 2 shows the space-dependent current noise autocovariance function computed for this device. Several comments are in order: a) The maximum value of $\sigma^{2}$, the noise power, occurs at the $0.15 \mu \mathrm{m}$ point in which the electrostatic field reaches its maximum value. This result had already been observed for Bulk silicon in [2], b) The auto-covariance function remains positive for points in the middle of the device for which the

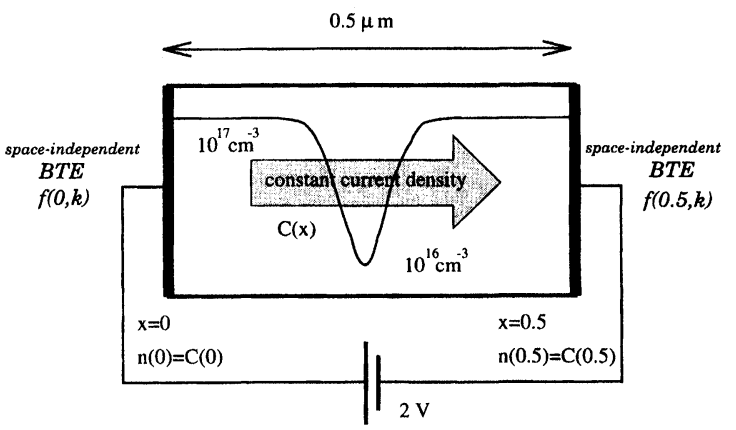

FIGURE 1 shows the device considered in our simulations. The device is $0.5 \mu \mathrm{m}$ long, the applied potential is equal to 2 volts, the doping profile is Gaussian and symmetrical with its lowest value in the middle of the device.

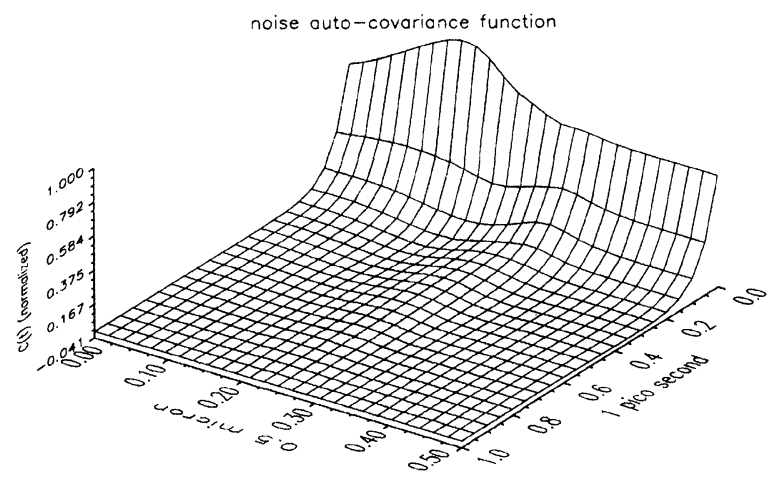

FIGURE 2 shows the current longitudinal noise autocorrelation function corresponding to our simulations. The value of $\sigma^{2}$ reaches it maximun value at $0.15 \mu \mathrm{m}$, point for which the electrostatic field has reached its maximum value. 


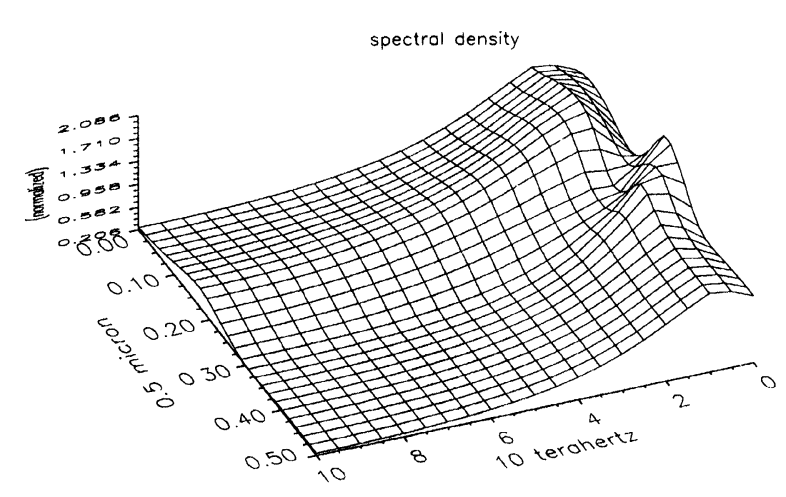

FIGURE 3 shows the spectral density function corresponding to Figure 2. This figure shows that the noise at $0.15 \mu \mathrm{m}$, point for which the electrostatic field has reached its maximum value, has important high frequency components.

doping reaches its minimum and c) the autocovariance functions at both ends are identical, a result that is in complete agreement with the assumed space-independence condition for the boundaries of the device. Figure 3 shows the corresponding spectral density function to Figure 2. The most important feature of this figure is that higher frequency components are observed again for the points in the area of large electrotatic field.

\section{CONCLUSIONS}

Numerical results for the space-dependent autocovariance and spectral density function of noise due to acoustic and optical phonon scattering were presented. A simple algorithm to compute the noise auto-correlation function that accounts for the effect of the spatial variation of the doping was described. The algorithm was succesfully employed for noise calculations for a sub-micron stucture in stationary regime. Hence the SDE noise model is demonstrated to be a viable approach for space-dependent noise computations .

\section{References}

[1] Korman, C. E. and Mayergoyz, I. D. (1996). "Semiconductor Noise in the Framework of Semiclassical Transport", Phys. Rev. B, 54, 24.
[2] Piazza, Alfredo J. and Korman, C. E., "Computation of the Spectral Density of Current Fluctuations in Bulk Silicon Based on the Solution of the Boltzmann Transport Equation", accepted for publication.

[3] Hongchin, Lin, Neil, Goldsman and Mayergoyz, I. D. (1992). "Device Modeling by Deterministic Self-Consistent Solution of Poisson and Boltzmann Transport Equations", Solid-St. Electron, 35(6), pp. 769-778.

\section{Authors' Biographies}

Can E. Korman was born in Silver Spring, Maryland. He received the B.S., M.S. and Ph.D. degrees in Electrical Engineering from the University of Maryland in 1985, 1987 and 1990, respectively. In 1991, he joined the Department of Electrical Engineering and Computer Science at the George Washington University, Washington, DC where he is currently an Assistant Professor.

His current research is in the area of numerical simulation of semiconductor devices, semiconductor noise characterization, parallel algorithms, stochastic differential equations, VLSI and magnetic viscosity. He is the recipient of the National Science Foundation Research Initiation Award. He has also received the following teaching awards at the George Washington University: the Eta Kappa $\mathrm{Nu}$ Association Teacher of the Year Award for 1993-94 and the Engineer's Council Faculty of the Year Award for 1994-95. He is a member of the IEEE Electron Devices Society and the Circuits and Systems Society.

Alfredo Piazza was born in Lima, Peru, in 1963. $\mathrm{He}$ received the B.S. degree in industrial engineering from the Pontificia Universidad Catolica del Peru in 1988. In 1995 he obtained the M.S. degree in electrical engineering with concentration in electrophysics from the George Washington University. He continues toward the Ph.D. in electrical engineering with concentration in systems science, networks and controls. His research concentrates in the numerical simulation of semiconductor devices. His current research is on the topic of noise characterization of semiconductor materials by means of the direct solution of the Boltzmann Transport Equation. 

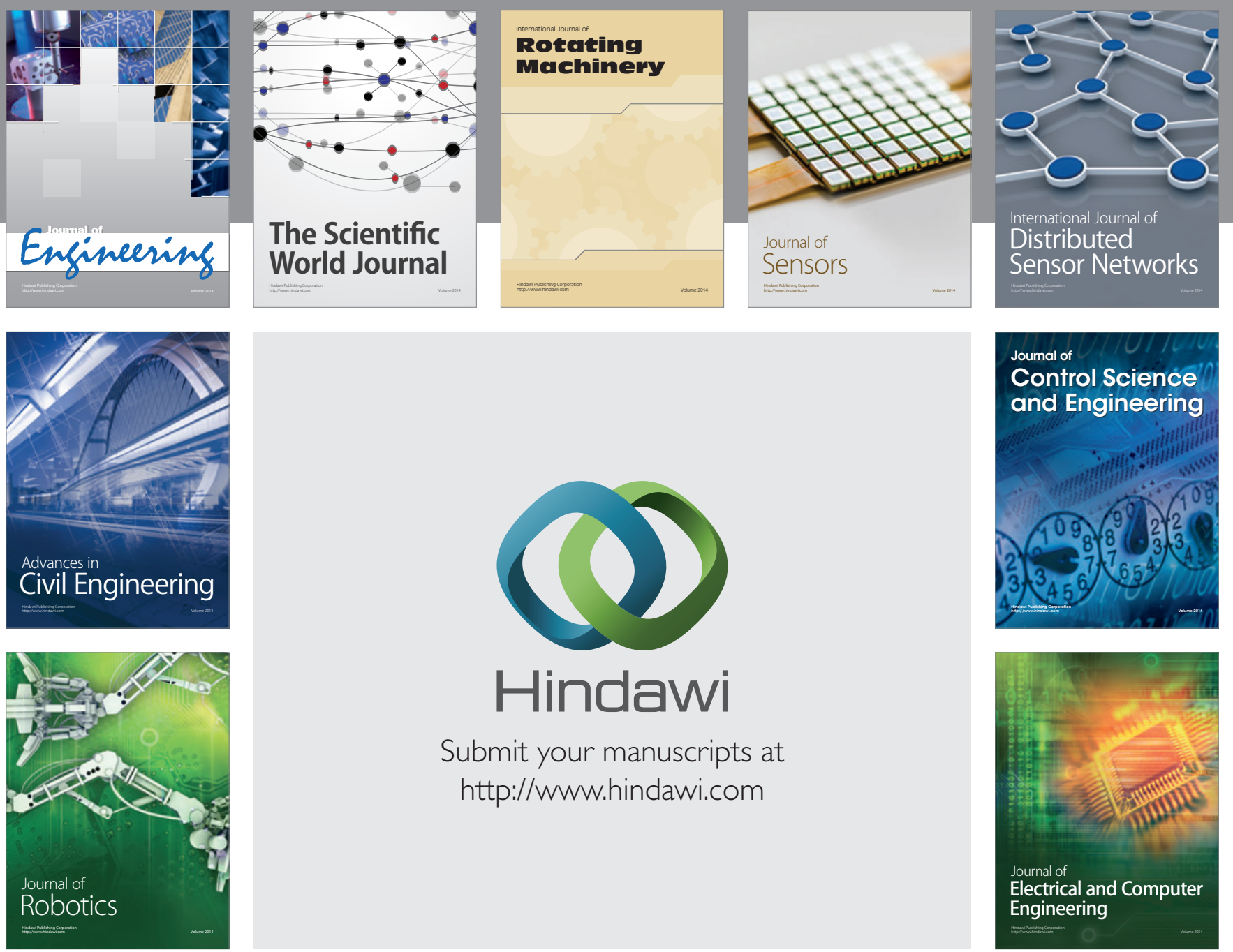

Submit your manuscripts at

http://www.hindawi.com
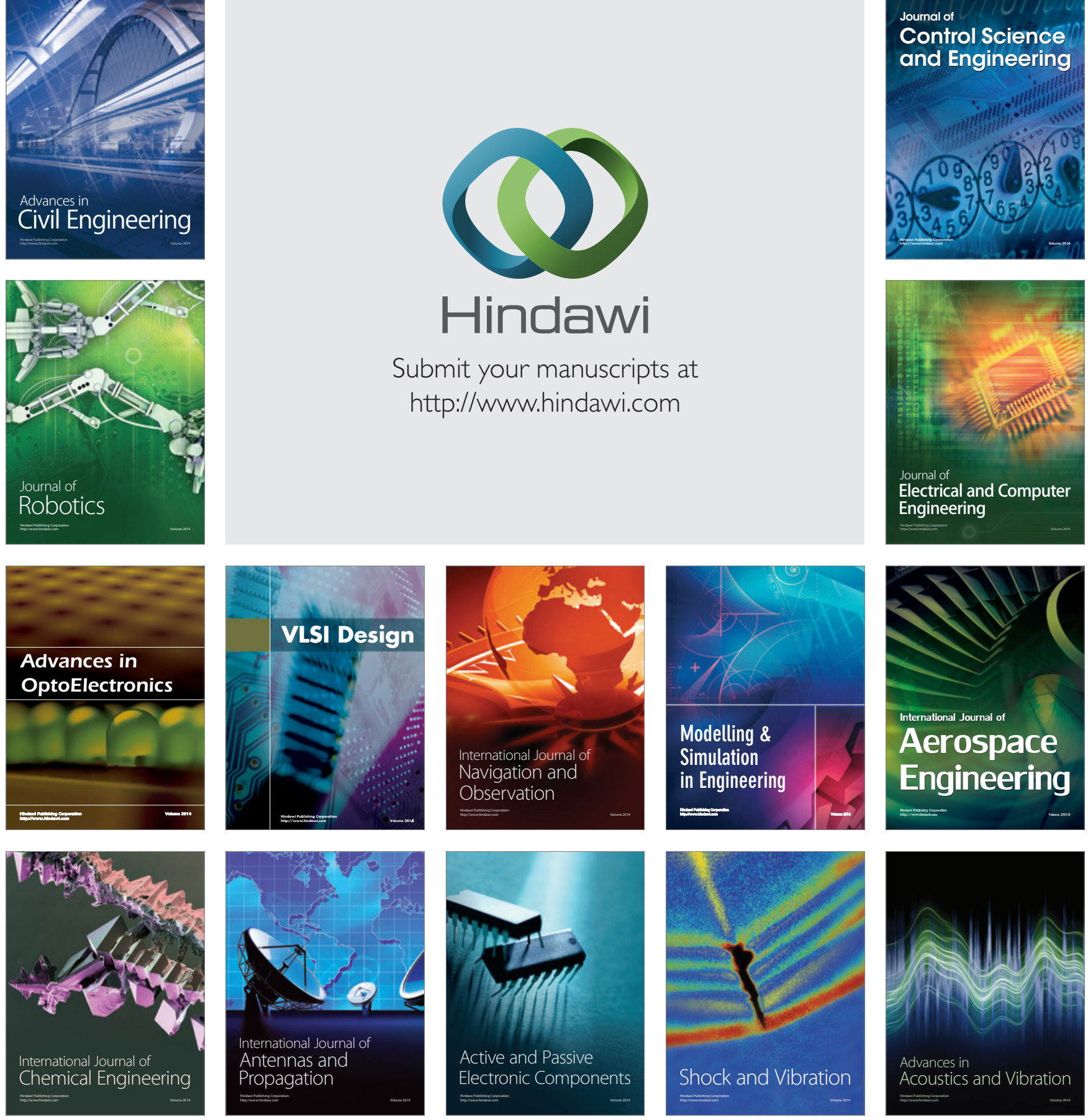